\title{
Steady-state first-pass perfusion (SSFPP): A 3D TWIST in myocardial first-pass perfusion imaging
}

\author{
Shivraman Giri ${ }^{1 *}$, Hui Xue ${ }^{2}$, Abdul Wattar ${ }^{1}$, Yu Ding ${ }^{1}$, Randall M Kroeker ${ }^{3}$, Gerhard Laub ${ }^{4}$, Peter Kellman ${ }^{6}$, \\ Sven Zuehlsdorff, Subha V Raman ${ }^{1}$, Orlando P Simonetti ${ }^{1}$ \\ From 15th Annual SCMR Scientific Sessions \\ Orlando, FL, USA. 2-5 February 2012
}

\section{Summary}

A new approach to myocardial first-pass perfusion imaging is presented; this technique, called SSFPP, is based on 3D SSFP sequence whereby magnetization is maintained in constant steady-state, while data acquisition is gated to diastole. This results in high SNR and CNR, and other characteristics that can potentially mitigate dark rim artifacts. The high-blood myocardial contrast of SSFP allows automatic segmentation, which, combined with registration, facilitates image analysis.

\section{Background}

Although introduced in 1990, myocardial first-pass perfusion imaging has not yet become a routine diagnostic tool, primarily because of insufficient image quality, insufficient coverage, and dark rim artifacts (DRA). Current techniques rely on a saturation recovery (SR) preparation for T1 contrast, resulting in poor SNR, low efficiency, and k-space modulation during SR. Further, the post-processing of these images is tedious. In this work, we propose an alternative perfusion imaging technique, called Steady-State First Pass Perfusion (SSFPP).

\section{Objective}

To develop a new 3D first-pass perfusion imaging technique that can potentially address the limitations of current methods.

\section{Theory}

SSFPP is a 3D SSFP sequence in which the magnetization is maintained in constant steady-state while the data acquisition is gated to diastole. The SNR and CNR are similar to those in SSFP cine imaging, allowing the

\footnotetext{
${ }^{1}$ The Ohio State University, Columbus, OH, USA

Full list of author information is available at the end of the article
}

use of automatic segmentation algorithms. Furthermore, the tissue contrast is dependent on $\mathrm{T} 1 / \mathrm{T} 2$; serendipitously, this causes blood signal to remain almost constant, whereas the myocardial signal exhibits a nearly linear correlation with contrast agent concentration. Maintenance of steady-state throughout data acquisition avoids $\mathrm{k}$-space modulation, and the elimination of saturation recovery time increases data acquisition efficiency by reducing deadtime.

\section{Methods}

SSFPP was implemented on a $1.5 \mathrm{~T}$ scanner (Avanto, Siemens). RF pulse (time-bandwidth product $=10$, flip angle $\sim 40^{\circ}$.) was optimized for 3D slab excitation profile. Other parameters: resolution $\sim 2.2 \times 2.8 \times 8 \mathrm{~mm}^{3}$, matrix $160 \times 103 \times 6$, slab oversampling $33.3 \%$, TR $=\sim 2.7 \mathrm{~ms}$, Multihance $(0.1 \mathrm{mmol} / \mathrm{kg})$. 3D K-space was acquired using parallel imaging (GRAPPA, rate $=3,24$ intrinsic reference lines, 32 channel phased array coil (QED LLC)) and TWIST acquisition scheme; for the latter, a central $4 \%$ of $\mathrm{k}$-space was updated every frame, whereas the peripheral region was undersampled at $33 \%$, leaving a "temporal footprint" of 3 heart beats. Acquisition time per 3D frame was $\sim 300-340 \mathrm{~ms}$. Images were acquired in three healthy subjects during contrast agent injection to evaluate feasibility of this new method.

Non-rigid registration, optimized for dynamically varying contrast, was used for three-dimensional motioncorrection prior to automated contouring of endo and epicardial borders.

Pixel-wise contrast enhancement ratio (CER) maps were computed for each frame, where each pixel is given by: $\left(S_{n}-S_{\text {baseline }}\right) / S_{\text {baseline; }}$ time intensity curves (TIC) of these CER images were used for semi-quantitative analysis. 

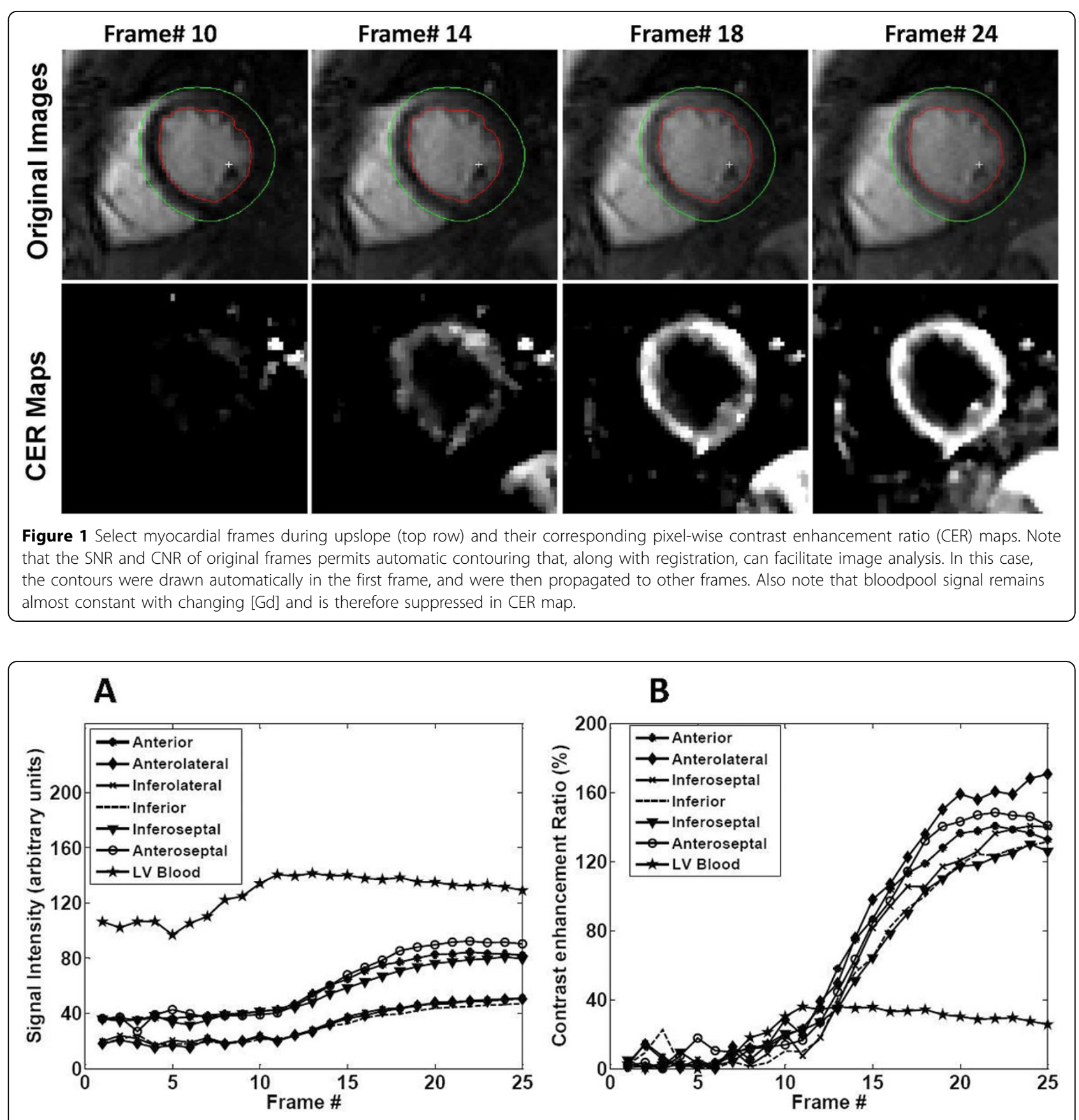

Figure 2 Signal intensity (A) and contrast enhancement ratio (CER) (B) curves for left ventricular blood pool and six myocardial segments for the slice shown in Figure 1. Note that the blood signal stays nearly constant compared with myocardium. Also note that the signal intensity is affected by the use of phase array coils, with the segments closer to the coil having higher signal; this is partly corrected for in the CER maps.

\section{Results}

Figures 1 and 2 show images and TICs from a SAX slice of one subject. Similar results were noted in other two subjects.

\section{Conclusions}

3D SSFPP avoids many of the suspected causes of DRA and could potentially mitigate this problem. Steady-state imaging provides high SNR and CNR that, combined 
with image registration, can facilitate effective perfusion quantification.

\section{Funding}

The project is partially supported by Award Number R01HL102450 from the National Heart, Lung, and Blood Institute.

Author details

${ }^{1}$ The Ohio State University, Columbus, OH, USA. ${ }^{2}$ Siemens Corporate Research, Princeton, NJ, USA. ${ }^{3}$ Siemens Healthcare, Winnipeg, MB, Canada.

${ }^{4}$ Siemens Healthcare, San Francisco, CA, USA. ${ }^{5}$ Siemens Healthcare, Chicago,

IL, USA. ${ }^{6}$ National Institutes of Health, Bethesda, MD, USA.

Published: 1 February 2012

doi:10.1186/1532-429X-14-S1-P251

Cite this article as: Giri et al:: Steady-state first-pass perfusion (SSFPP): A

3D TWIST in myocardial first-pass perfusion imaging. Journal of

Cardiovascular Magnetic Resonance 2012 14(Suppl 1):P251.

Submit your next manuscript to BioMed Central and take full advantage of:

- Convenient online submission

- Thorough peer review

- No space constraints or color figure charges

- Immediate publication on acceptance

- Inclusion in PubMed, CAS, Scopus and Google Scholar

- Research which is freely available for redistribution

Submit your manuscript at www.biomedcentral.com/submit
C Biomed Central 